\title{
Non iRECIST Complete Response-Non iRECIST Unconfirmed Progressive Disease
}

National Cancer Institute

\section{Source}

National Cancer Institute. Non iRECIST Complete Response-Non iRECIST Unconfirmed

Progressive Disease. NCI Thesaurus. Code C142361.

Persistence of one or more non-target lesion(s) without unequivocal progression

following an initial apparent radiographic progression. 